\section{(6) OPEN ACCESS}

\title{
Survival of patients with small cell lung cancer undergoing lung resection in England, 1998-2009
}

\author{
Margreet Lüchtenborg, ${ }^{1,2}$ Sharma P Riaz, ${ }^{2}$ Eric Lim, ${ }^{3}$ Richard Page, ${ }^{4}$ David R Baldwin, ${ }^{5}$ \\ Erik Jakobsen, ${ }^{6}$ Peter Vedsted, ${ }^{7}$ Mike Lind, ${ }^{8}$ Michael D Peake, ${ }^{9}$ Anders Mellemgaard, ${ }^{10}$ \\ James Spicer, ${ }^{11}$ Loic Lang-Lazdunski, ${ }^{12}$ Henrik Møller ${ }^{1,7}$
}

For numbered affiliations see end of article.

\section{Correspondence to}

Professor Henrik Møller, King's Health Partners Cancer Centre, King's College London, Research Oncology, Bermondsey Wing, 3rd Floor, Guy's Hospital, Great Maze Pond, London SE1 9RT, UK; henrik.moller@kcl.ac.uk

Received 16 May 2013 Revised 3 October 2013 Accepted 8 October 2013 Published Online First 30 October 2013

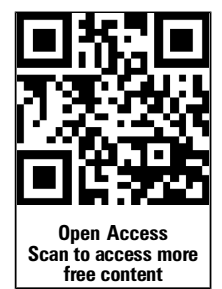

To cite: Lüchtenborg $M$, Riaz SP, Lim E, et al. Thorax 2014;69:269-273.

\section{ABSTRACT}

Introduction Chemotherapy or chemoradiotherapy is the recommended treatment for small cell lung cancer (SCLC), except in stage I disease where clinical guidelines state there may be a role for surgery based on favourable outcomes in case series. Evidence supporting adjuvant chemotherapy in resected SCLC is limited but this is widely offered.

Methods Data on 359873 patients who were diagnosed with a first primary lung cancer in England between 1998 and 2009 were grouped according to histology (SCLC or non-SCLC (NSCLC)) and whether they underwent a surgical resection. We explored their survival using Kaplan-Meier analysis and Cox regression, adjusting for age, sex, comorbidity and socioeconomic status.

Results The survival of 465 patients with resected SCLC was lower than patients with resected NSCLC (5-year survival $31 \%$ and $45 \%$, respectively), but much higher than patients of either group who were not resected (3\%). The difference between resected SCLC and NSCLC diminished with time after surgery. Survival was superior for the subgroup of 198 'elective' SCLC cases where the diagnosis was most likely known before resection than for the subgroup of 267 'incidental' cases where the SCLC diagnosis was likely to have been made after resection.

Conclusions These data serve as a natural experiment testing the survival after surgical management of SCLC according to NSCLC principles. Patients with SCLC treated surgically for early stage disease may have survival outcomes that approach those of NSCLC, supporting the emerging clinical practice of offering surgical resection to selected patients with SCLC.

\section{INTRODUCTION}

The well-established division of primary malignant tumours of the lung into small cell lung cancer (SCLC) and non-SCLC (NSCLC) is due to the clear difference in presentation and behaviour of the two groups. ${ }^{1}$ SCLC proliferates more rapidly and has a high propensity to metastasise. It is more responsive to first-line chemotherapy. Patients with SCLC usually present with locally advanced or metastatic disease because of the high mitotic index of this disease and its propensity to early systemic metastasis. In contrast, between $10 \%$ and $20 \%$ of patients with NSCLC present with early stage disease (I or II) and are potential candidates for surgical resection.

\section{Key messages}

What is the key question?

- What would be the survival outcome in patients with SCLC if they were managed surgically like patients with NSCLC?

\section{What is the bottom line?}

- Despite the inherent poor prognosis of SCLC, surgically resected patients with SCLC have 5 -year survival of $31 \%$ compared with $45 \%$ in resected NSCLC and $3 \%$ in patients with unresected lung cancer of either subtype.

\section{Why read on?}

- This is a nationally based clinical follow-up study of 359873 patients with lung cancer of whom a large series of 465 were patients who were resected with SCLC. The results support the emerging clinical practice of offering surgical resection to selected patients with SCLC.

Surgery is inappropriate for the majority of patients with SCLC who present with locally advanced or metastatic disease. These are treated with chemotherapy (and often with thoracic and prophylactic cranial radiotherapy), which is associated with a high response rate but which is often followed by subsequent relapse and eventual death. Nevertheless, on rare occasions patients are identified with SCLC histology and with early stage disease potentially suitable for resection. ${ }^{2}$ In these situations surgery may be offered, but with little evidence available to guide the treating thoracic surgeons and oncologists. Combination chemoradiotherapy in highly selected fit patients with limited stage disease has been reported to result in 5 -year survival of up to $26 \% .^{3}$

The MRC-UK randomised study published in $1973^{4}$ showed that surgery made outcomes worse for patients with SCLC and led to firm ideas against surgery among clinicians for the next two decades. In the intervening years, significant advances have been made in the staging of lung cancer with CT and, more recently, positron emission tomography (PET) imaging. Recently there have been a number of reports of patients being treated with surgery for SCLC with apparently 
good results. ${ }^{5-7}$ The clinical relevance of these studies is limited by the lack of an appropriate control group and small numbers of patients treated over periods of many years. ${ }^{8}$ Furthermore, many patients in these reports had a diagnosis of SCLC made only at postoperative examination of the resection specimen so that the working diagnosis at the time of decision to offer surgery was NSCLC. ${ }^{9}$

We examined the lung cancer data in the National Cancer Data Repository (NCDR), which includes information on surgical treatment. The primary outcome was survival of patients with SCLC who underwent surgery in comparison with patients with NSCLC, and specifically in the context of surgery taking place after the diagnosis of SCLC (elective cases) or the diagnosis of SCLC being established subsequent to surgical resection (incidental cases).

\section{METHODS}

\section{Patient data}

Information on 383027 lung cancer diagnoses (ICD10 C33C34) in England between 1998 and 2009 was retrieved from the NCDR. Cancers identified from a death certificate only (20 778; 5\%) were excluded. We included only records related to the patients' first primary lung cancer, leading to the exclusion of 1421 tumour records. We also excluded 955 patients without a valid NHS number because they could not be linked to the hospital episode statistics (HES) data. The analyses presented here thus include 359873 patients with a first primary lung cancer (table 1).

Tumours were grouped according to the ICDO2 classification. SCLC was defined by morphology codes 8041-8045. All remaining codes, including cancers with unspecified histology (34\% of all cases; $5 \%$ of resected NSCLC cases) were classified as NSCLC.

Information on surgical resection was retrieved from the linked HES records. Types of surgical resections included were as previously defined ${ }^{10}$ : lobectomy or bilobectomy (66\%), partial lobectomy or wedge resection (15\%), pneumonectomy $(15 \%)$, sleeve resection $(1 \%)$ and other less common procedures (other or unspecified excisions of (lesions of) trachea, carina, lung and chest wall, 4\%). Data on surgery from 1 month before to 6 months after the date of diagnosis were included. If patients had more than one record of a relevant surgical procedure, the first procedure was used in the analysis. There was no information available on tumour stage.

Socioeconomic deprivation was measured by lower super output area of residence (each comprising a population of around 1500 people) based on the income domain of the Indices of Deprivation 2004, 2007, 2010, relevant to the year of diagnosis and grouped into quintiles. Each patient was thus assigned to a socioeconomic quintile based on their postcode of residence at the time of the cancer diagnosis.

Table 1 Lung cancer registrations in England 1998-2009

\begin{tabular}{|c|c|c|c|c|}
\hline \multirow{2}{*}{ Histology } & \multicolumn{4}{|l|}{ Surgery } \\
\hline & No & Yes & Yes (\%) & Total \\
\hline NSCLC & 284355 & 29670 & 9 & 314025 \\
\hline SCLC & 45383 & 465 & 1 & 45848 \\
\hline SCLC (\%) & 14 & 2 & & 13 \\
\hline Total & 329738 & 30135 & 8 & 359873 \\
\hline
\end{tabular}

For each patient, comorbidity information was obtained using diagnosis codes recorded in the HES. All diagnoses from 2 years before to 3 months after the patient's date of diagnosis were classified according to the scores from the weighted Charlson comorbidity index ${ }^{11}$ and modified to exclude cancer itself as a comorbid condition. The resulting scores were aggregated into categories of increasing severity of comorbidity.

The cancer registration record included a date of diagnosis, preferably taken as the date of sampling of the tissue that gave rise to the histological diagnosis. By considering the date of diagnosis and the date of the surgical resection, we attempted to further classify the resected SCLC into those (elective) cases that were known (typically from an earlier diagnostic biopsy) to be SCLC before the surgical resection was planned, and those (incidental) cases that were diagnosed as SCLC on the basis of the examination of the resection specimen. We considered those with a resection on day 8 after the SCLC diagnosis or later to be predominantly elective cases $(n=198$; table 2$)$. When the resection was earlier than the date of diagnosis or near the date of diagnosis (range from 28 days before the diagnosis to 7 days after), we considered it most likely that the diagnosis was established as SCLC on the basis of the resected tissue $(\mathrm{n}=267)$.

In a small minority of cases of resection taking place before the diagnosis, this sequence of events occurs because the date of diagnosis was taken to be the date of the pathology report instead of the date of tissue sampling which may have occurred earlier. These date sequences are not routinely quality assured and we made no attempt to correct the very few outlying values.

\section{Data analysis}

Survival functions curves were estimated for each group using the Kaplan-Meier method and corresponding 5-year survival estimates with $95 \%$ CI (corresponding to a $\mathrm{p}$ value criterion of 5\%) were calculated.

Univariate and multivariate Cox proportional hazards analyses were performed to assess the all-cause mortality HRs according to histological type and surgery, and adjusted for age, sex, comorbidity and socioeconomic deprivation. These adjustments were decided a priori.

Survival time was calculated from the date of surgery for those patients undergoing surgical resection or date of diagnosis for those who did not, until date of death from any cause. The remaining patients were censored at the end of follow-up on 31 December 2010. The Cox regression analyses were divided into three prespecified intervals of follow-up time: first month of follow-up, intermediate period from 1 month to 1 year, and long-term follow-up (1 year and longer).

All analyses were performed using Stata V.11.0 (StataCorp LP, College Station, Texas, USA).

Table 2 Interval from diagnosis to operation in 465 patients with SCLC with surgical resection

\begin{tabular}{lcrl}
\hline Days & Frequency & $\%$ & Subgroup \\
\hline-28 to -8 & 4 & 1 & Incidental \\
-7 to -2 & 14 & 3 & Incidental \\
-1 to 1 & 236 & 51 & Incidental \\
2 to 7 & 13 & 3 & Incidental \\
8 to 176 & 198 & 43 & Elective \\
\hline
\end{tabular}


Figure 1 Kaplan-Meier survival analysis of resected and unresected patients with non-small cell lung cancer (NSCLC) and small cell lung cancer (SCLC). For the resected patients, follow-up starts on the date of resection; for unresected patients it starts on the date of diagnosis.

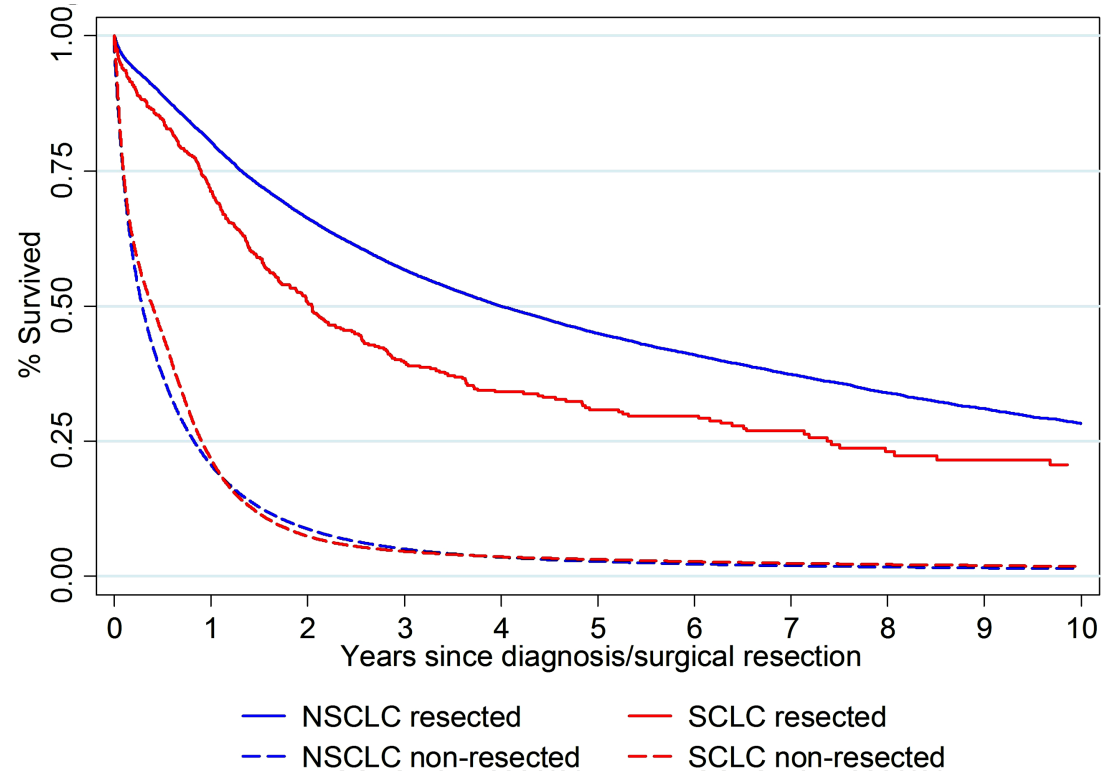

\section{RESULTS}

Of the 359873 lung cancer diagnoses during the 12-year period from 1998 to 2009, 45848 (13\%) were SCLC of which 465 ( $1 \%$ of all SCLC) were treated surgically (table 1). By comparison, of the 314025 patients with NSCLC, 29670 (9\%) underwent surgical resection. The survival functions for the four groups of patients are presented in figure 1. Five-year survival was superior for patients with NSCLC who underwent a surgical resection at $45 \%$ (95\% CI 44\% to 46\%). Five-year survival among patients with SCLC who underwent a surgical resection was lower at 31\% (95\% CI 26\% to 36\%), but considerably higher than the survival among unresected patients with NSCLC $(2.72 \%$ (95\% CI $2.66 \%$ to $2.79 \%)$ ) and those with unresected SCLC (3.08\% (95\% CI 2.91\% to 3.26\%)).

Figure 2 shows the further subclassification of resected SCLC cases depending on the time between diagnosis and resection. The subgroup dominated by an incidental SCLC diagnosis (green survival function) had lower survival than the subgroup dominated by an elective resection of known SCLC (red survival function), but the two survival functions were similar and converged from the third year of follow-up onwards.

Table 3 shows the results of multivariate Cox regression analysis comparing the survival of all five groups of lung cancer patients. Compared with the baseline group of resected NSCLC, the survival of unresected groups with either histology was inferior throughout the period of follow-up.

In the first month of follow-up, survival of cases of electively resected SCLC were identical to cases of the resected NSCLC (mortality $\mathrm{HR}=0.98 ; 95 \%$ CI 0.46 to 2.05 ) but, in the longer, term these electively resected SCLC patients had higher death rates than resected NSCLC patients (1 month to 1 year: 1.36 (95\% CI 1.00 to 1.84 ); $\geq 1$ year: 1.47 (95 CI 1.18 to 1.82 )). The subgroup of incidentally resected SCLC cases initially had an HR of 2.24 compared with resected NSCLC cases but this excess decreased subsequently (1 month to 1 year: 1.65 (95\% CI 1.29 to 2.11 ); $\geq 1$ year: 1.38 (95\% CI 1.13 to 1.68$)$ ).

In the direct comparison of the two resected SCLC groups (elective and incidental), the difference was large and statistically
Figure 2 Kaplan-Meier survival analysis of resected and unresected patients with non-small cell lung cancer (NSCLC) and small cell lung cancer (SCLC). Resected SCLC cases stratified into elective and incidental cases as defined in table 2 . For the resected patients, follow-up starts on the date of resection; for unresected patients it starts on the date of diagnosis.

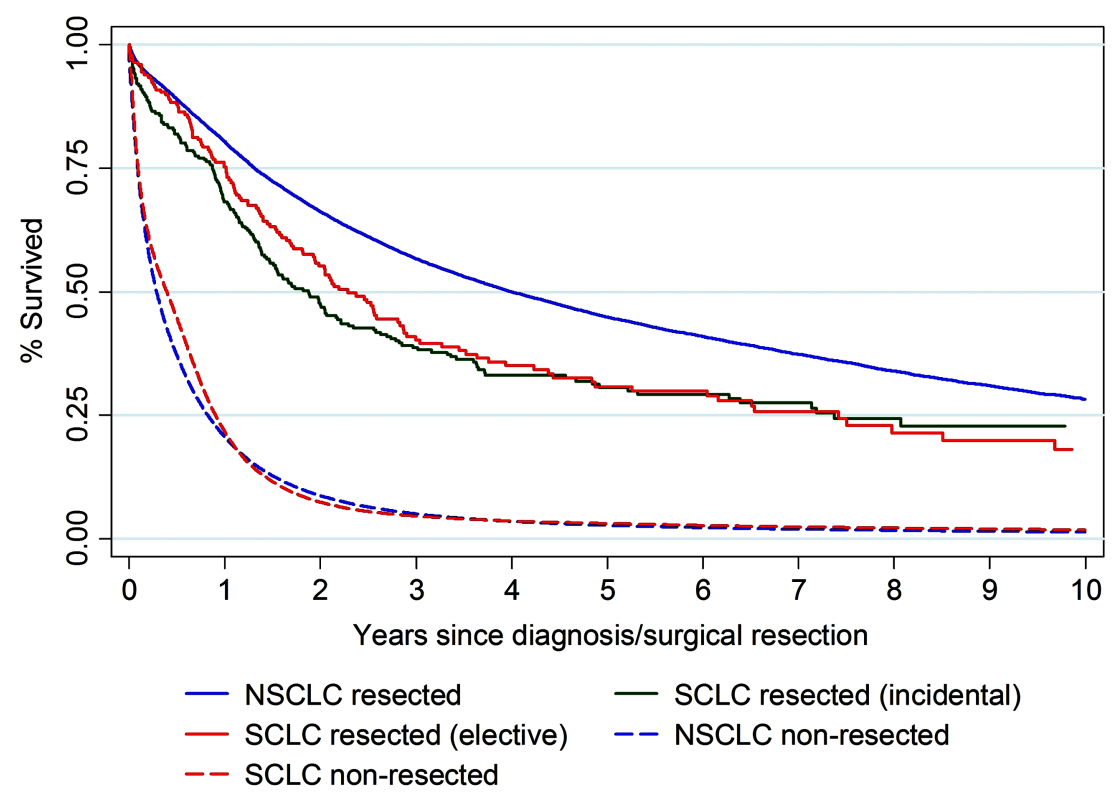


Table 3 Survival among 359873 lung cancer patients according to histological type and surgery

\begin{tabular}{|c|c|c|c|c|c|c|c|c|c|c|c|c|}
\hline \multirow[b]{2}{*}{ Period of follow-up: } & \multicolumn{4}{|c|}{ 0-1 Month } & \multicolumn{4}{|c|}{1 Month-1 Year } & \multicolumn{4}{|c|}{1 Year +} \\
\hline & HR & $95 \%$ & & $p$ Value & HR & $95 \% \mathrm{Cl}$ & & $\mathrm{p}$ Value & HR & $95 \% \mathrm{C}$ & & p Value \\
\hline NSCLC with surgery (29 670) & 1.00 & & & & 1.00 & & & & 1.00 & & & \\
\hline SCLC with surgery (incidental) $(267)^{*}$ & 2.24 & 1.45 & 3.45 & $<0.001$ & 1.65 & 1.29 & 2.11 & $<0.001$ & 1.38 & 1.13 & 1.68 & 0.002 \\
\hline SCLC with surgery (elective) (198) $\dagger$ & 0.98 & 0.46 & 2.05 & 0.949 & 1.36 & 1.00 & 1.84 & 0.050 & 1.47 & 1.18 & 1.82 & 0.001 \\
\hline NSCLC, no surgery (284 355) & 5.59 & 5.26 & 5.94 & $<0.001$ & 6.76 & 6.57 & 6.96 & $<0.001$ & 3.30 & 3.23 & 3.37 & $<0.001$ \\
\hline SCLC, no surgery (45 383) & 6.26 & 5.88 & 6.67 & $<0.001$ & 6.59 & 6.39 & 6.80 & $<0.001$ & 3.89 & 3.77 & 4.00 & $<0.001$ \\
\hline
\end{tabular}

significant in the first month but the HRs were more similar and not statistically significantly different after 1 month of follow-up. The estimated HRs were generally not sensitive to the statistical adjustment for comorbidity and socioeconomic deprivation.

Of the resected SCLC patients, 275 (59\%) had a lobectomy or bilobectomy and 146 (31\%) had a pneumonectomy. The hazard of death was overall higher in patients with pneumonectomy (adjusted HR 1.53 ; 95\% CI 1.05 to 2.25 ). Forty-four (9\%) of the patients had other operations and had an intermediate hazard of death (HR 1.39; 95\% CI 1.08 to 1.78).

\section{DISCUSSION}

The results of our analysis of the English NCDR data on outcomes for surgery in SCLC suggest long-term survival outcomes approaching those in resected NSCLC, especially for the cohort of patients with SCLC who underwent elective resection. We deduce that surgery was elective in those cases in which histological diagnosis was determined in advance of surgery. Comparison of these results with other studies is difficult because the literature in this area is based mostly on small single institution series. ${ }^{7}$ One other population-based study based on Surveillance, Epidemiology and End Results (SEER) program registry data showed a comparable five-year survival for patients undergoing surgery for SCLC of about $40 \% .^{5}$

Case selection may have contributed to the differences we report in outcomes for elective versus incidental surgery for SCLC. It is plausible that patients with known SCLC were more intensively worked up, with surgery offered only to those with very early stage, few comorbidities and good performance status. This could distort a comparison against outcome in patients who underwent surgery for NSCLC. Patients with NSCLC might be expected to have been offered surgery despite a relatively more advanced stage (including patients with stage IIIA) and more significant comorbidities than those with SCLC because surgery is a more accepted treatment modality in patients with NSCLC. For these reasons it is difficult to make clear comparative inferences on survival between resected SCLC and NSCLC in the absence of precise information on stage. Nevertheless, we report five-year overall survival of $31 \%$ in patients undergoing resection for SCLC. Perhaps the most pertinent comparison would be against those patients with a similar stage and comorbidity undergoing medical treatment.

A major limitation of the present study is the lack of information on stage and the inability to undertake a stage-adjusted comparison. This is because stage was not collected systematically by the cancer registries in this period, and the 6th edition of the TNM classification did not specify $\mathrm{T}, \mathrm{N}$ and $\mathrm{M}$ dimensions for
SCLC but by classification of limited or extensive disease. ${ }^{1}{ }^{12}$ Data from the US SEER registry, however, did report better outcomes in patients with SCLC who underwent surgery compared with medical treatment, both in stage I and stage II. ${ }^{5}$

A further limitation is the absence of information on treatments other than surgery. The NCDR data that we used for this study do not yet include information on radiotherapy and chemotherapy. It is planned to amend these data in coming versions, and it will be especially relevant then to compare the survival of resected SCLC patients with that of patients with SCLC on other lines of active therapy such as combined chemoradiotherapy.

It is possible that, among SCLCs, some tumours have a more favourable growth pattern and less aggressive tumour biology. Incidentally resected patients with SCLC usually present with a small peripheral nodule without hilar or mediastinal lymphadenopathy. This clinical presentation is unusual as SCLC usually presents with advanced or locally advanced disease. Although variations in tumour biology have not been reported, they may account for the marked variation in clinical presentation and outcome among patients with SCLC.

Published guidelines are not fully consistent on recommendations concerning the surgical management of SCLC, ${ }^{13-15}$ reflecting the paucity of evidence in this area.

The difference between survival in resected and non-resected SCLC patients may seem impressive, but there is strong selection of patients for surgery and this comparison is biased by stage and other case mix variables so it is not a fair representation of the efficacy of surgical resection in SCLC. The more relevant comparison is between resected SCLC patients in the incidental subgroup and resected NSCLC patients. This contrast addresses the relevant clinical question: "What would be the survival outcome in SCLC patients if they were managed surgically like NSCLC patients?" Comparison of the available case mix variables between these two groups (sex, age, socioeconomic status, comorbidity and ethnicity) did not reveal any important differences, so the natural experiment is not confounded by those factors.

Data have been continuously gathering in the last few years in support of the role of surgery for SCLC but, in the absence of clinical trial evidence, the uptake for surgery is likely to remain low and patchy.

Previous major randomised trials of surgery for SCLC were performed in the era prior to PET, with clinical staging and preoperative selection based on either plain chest film ${ }^{4}$ or CT scanning, ${ }^{16}$ which in part accounts for the high proportion of exploratory thoracotomies by the inclusion of patients not currently accepted to be suitable for surgery. Many surgical series 
in selected patients report excellent survival; however, there is no available evidence on the prognosis of a similar group of patients treated with the current standard of concurrent chemoradiotherapy. The timing and need for a clinical trial has been emphasised internationally. ${ }^{17}{ }^{15}$ The purpose of such a study would be to evaluate progression-free survival comparing surgery with adjuvant chemotherapy versus concurrent chemoradiotherapy (ie, comparison of surgery against radiotherapy for local control). There is likely to be strong opinions as to the stage where clinical equipoise exists and, if so, pragmatic multidisciplinary team-based randomisation can perhaps be considered, as per the design of PulMiCC, a randomised clinical trial comparing surgery versus no surgery for the treatment of colorectal metastases to the lung. ${ }^{18}$

\section{CONCLUSION}

This nationally based cohort study of outcomes of surgery for SCLC suggests good results may be obtained, despite the inherent poor prognosis of SCLC. Carefully selected patients with SCLC treated with surgery may have survival outcomes that approach those of NSCLC. We suggest that any lung cancer patient fit for treatment should undergo full staging procedures and that resection should be offered to patients with early stage SCLC based on similar principles to those for NSCLC.

\footnotetext{
Author affiliations

${ }^{1}$ Cancer Epidemiology and Population Health, King's Health Partners Cancer Centre, London, UK

${ }^{2}$ Public Health England, Knowledge \& Intelligence Team, London, UK

${ }^{3}$ The Academic Division of Thoracic Surgery, The Royal Brompton Hospital, London, UK

${ }^{4}$ Department of Thoracic Surgery, Liverpool Heart and Chest Hospital, Liverpool, UK ${ }^{5}$ Respiratory Medicine Unit, Nottingham University Hospitals and University of Nottingham, David Evans Centre, Nottingham City Hospital Campus, Nottingham, UK

${ }^{6}$ Department of Thoracic Surgery, The Danish Lung Cancer Registry, Odense University Hospital, Odense, Denmark

${ }^{7}$ Department of Public Health and Research Centre for Cancer Diagnosis in Primary Care, Aarhus University, Aarhus, Denmark

${ }^{8}$ Queens Centre for Oncology and Haematology, Hull and East Yorkshire NHS Trust, Hull, UK

${ }^{9}$ Department of Respiratory Medicine, Glenfield Hospital, Leicester, UK

${ }^{10}$ Department of Oncology, Herlev University Hospital, Copenhagen, Denmark

${ }^{11}$ Department of Research Oncology, Division of Cancer Studies, King's College London, London, UK

${ }^{12}$ Department of Thoracic Surgery, Guy's and St Thomas' NHS Foundation Trust, and Division of Cancer Studies, King's College London, London, UK
}

Acknowledgements This paper is a contribution from the National Cancer Intelligence Network and is based on the information collected and quality assured by the national cancer registration service in England (http://www.ukacr.org; http:// www.ncin.org.uk). The research was supported by the National Institute for Health Research (NIHR) Biomedical Research Centre based at Guy's and St Thomas' NHS Foundation Trust and King's College London. The views expressed are those of the authors and not necessarily those of the NHS or the NIHR.

Contributors HM and ML designed the study and led the editorial process. SPR did the statistical analysis, supported by HM and ML. ML, HM, EL, RP, DRB, AM and LL-L provided paragraphs of text, and all authors contributed to the manuscript by correcting and revising consecutive versions of the manuscript. All authors hereby contributed to the interpretation of the data and reviewed the manuscript for intellectual content, and they all approved the final version.

\section{Competing interests None.}

Provenance and peer review Not commissioned; externally peer reviewed.

Open Access This is an Open Access article distributed in accordance with the Creative Commons Attribution Non Commercial (CC BY-NC 3.0) license, which permits others to distribute, remix, adapt, build upon this work non-commercially, and license their derivative works on different terms, provided the original work is properly cited and the use is non-commercial. See: http://creativecommons.org/ licenses/by-nc/3.0/

\section{REFERENCES}

1 Sobin LH, Wittekind C. International Union Against Cancer (UICC) TNM classification of malignant tumors. 6th edn. New York: Wiley-Liss, 2002.

2 Baldwin DR, White B, Schmidt-Hansen M, et al.; Guideline Development Group. Diagnosis and treatment of lung cancer: summary of updated NICE guidance. BMJ 2011;342:d2110.

3 Turrisi AT 3rd, Kim K, Blum R, et al. Twice-daily compared with once-daily thoracic radiotherapy in limited small-cell lung cancer treated concurrently with cisplatin and etoposide. N Engl J Med 1999;340:265-71.

4 Fox W, Scadding JG. Medical Research Council comparative trial of surgery and radiotherapy for primary treatment of small-celled or oat-celled carcinoma of the bronchus. Lancet 1973;302:63-5.

5 Weksler B, Nason KS, Shende M, et al. Surgical resection should be considered for stage I and II small cell carcinoma of the lung. Ann Thorac Surg 2012;94:889-93.

6 Schreiber D, Rineer J, Weedon J, et al. Survival outcomes with the use of surgery in limited-stage small cell lung cancer: should its role be re-evaluated? Cancer 2010;116:1350-7.

7 Jones $C D$, Cummings IG, Shipolini AR, et al. Does surgery improve prognosis in patients with small-cell lung carcinoma? Interact Cardiovasc Thorac Surg 2013; 16:375-80

8 Lim E, Belcher E, Yap YK, et al. The role of surgery in the treatment of limited disease small cell lung cancer: time to re-evaluate. J Thorac Oncol 2008;3:1267-71.

9 Badzio A, Kurowski K, Karnicka-Mlodkowska H, et al. A retrospective comparative study of surgery followed by chemotherapy vs. non-surgical management in limited-disease small cell lung cancer. Eur J Cardiothorac Surg 2004;26:183-8.

10 Riaz SP, Linklater KM, Page R, et al. Recent trends in resection rates among non-small cell lung cancer patients in England. Thorax 2012;67:811-14.

11 Charlson ME, Pompei P, Ales KL, et al. A new method of classifying prognostic comorbidity in longitudinal studies: development and validation. J Chronic Dis 1987:40:373-83.

12 Shepherd FA, Ginsberg RJ, Haddad R, et al. Importance of clinical staging in limited small-cell lung cancer: a valuable system to separate prognostic subgroups. The University of Toronto Lung Oncology Group. J Clin Oncol 1993;11:1592-7.

13 Samson DJ, Seidenfeld J, Simon GR, et al. American College of Chest Physicians. Evidence for management of small cell lung cancer: ACCP evidence-based clinical practice guidelines (2nd edition). Chest 2007;132:314S-23S.

14 Lim E, Baldwin D, Beckles $M$, et al. Guidelines on the radical management of patients with lung cancer. Thorax 2010;65(Suppl 3):iii1-27.

15 National Institute for Health and Clinical Excellence. Lung cancer. The diagnosis and treatment of lung cancer. NICE Clinical Guideline 121. 2011.

16 Lad T, Piantadosi S, Thomas P, et al. A prospective randomized trial to determine the benefit of surgical resection of residual disease following response of small cell lung cancer to combination chemotherapy. Chest 1994;106:320S-3S.

17 Shepherd FA. Surgery for limited stage small cell lung cancer: time to fish or cut bait. J Thorac Oncol 2010;5:147-9.

18 Treasure T, Fallowfield L, Lees B. Pulmonary metastasectomy in colorectal cancer: the PulMiCC trial. J Thorac Oncol 2010;5:S203-6. 